\title{
Radiation hardness assessment of the charge-integrating hybrid pixel detector JUNGFRAU 1.0 for photon science
}

\author{
J. H. Jungmann-Smith, ${ }^{1, a), b)}$ A. Bergamaschi, ${ }^{1}$ M. Brückner, ${ }^{1}$ S. Cartier, ${ }^{1,2}$ R. Dinapoli, ${ }^{1}$ \\ D. Greiffenberg, ${ }^{1}$ A. Jaggi, ${ }^{1}$ D. Maliakal, ${ }^{1}$ D. Mayilyan, ${ }^{1}$ K. Medjoubi, ${ }^{3}$ D. Mezza, ${ }^{1}$ \\ A. Mozzanica, ${ }^{1}$ M. Ramilli, ${ }^{1}$ Ch. Ruder,${ }^{1}$ L. Schädler, ${ }^{1}$ B. Schmitt, ${ }^{1}$ X. Shi, ${ }^{1}$ and G. Tinti ${ }^{1}$ \\ ${ }^{1}$ Paul Scherrer Institute, 5232 Villigen PSI, Switzerland \\ ${ }^{2}$ Institute for Biomedical Engineering, University and ETHZ, 8092 Zürich, Switzerland \\ ${ }^{3}$ Synchrotron Soleil, L'Orme des Merisiers, Saint-Aubin-BP 48, 91192 GIF-sur-Yvette Cedex, France
}

(Received 16 October 2015; accepted 6 December 2015; published online 24 December 2015)

JUNGFRAU (adJUstiNg Gain detector FoR the Aramis User station) is a two-dimensional hybrid pixel detector for photon science applications in free electron lasers, particularly SwissFEL, and synchrotron light sources. JUNGFRAU is an automatic gain switching, charge-integrating detector which covers a dynamic range of more than $10^{4}$ photons of an energy of $12 \mathrm{keV}$ with a good linearity, uniformity of response, and spatial resolving power. The JUNGFRAU 1.0 application-specific integrated circuit (ASIC) features a $256 \times 256$ pixel matrix of $75 \times 75 \mu \mathrm{m}^{2}$ pixels and is bump-bonded to a $320 \mu \mathrm{m}$ thick Si sensor. Modules of $2 \times 4$ chips cover an area of about $4 \times 8 \mathrm{~cm}^{2}$. Readout rates in excess of $2 \mathrm{kHz}$ enable linear count rate capabilities of $20 \mathrm{MHz}$ (at $12 \mathrm{keV}$ ) and $50 \mathrm{MHz}$ (at $5 \mathrm{keV}$ ). The tolerance of JUNGFRAU to radiation is a key issue to guarantee several years of operation at free electron lasers and synchrotrons. The radiation hardness of JUNGFRAU 1.0 is tested with synchrotron radiation up to $10 \mathrm{MGy}$ of delivered dose. The effect of radiation-induced changes on the noise, baseline, gain, and gain switching is evaluated post-irradiation for both the ASIC and the hybridized assembly. The bare JUNGFRAU 1.0 chip can withstand doses as high as 10 MGy with minor changes to its noise and a reduction in the preamplifier gain. The hybridized assembly, in particular the sensor, is affected by the photon irradiation which mainly shows as an increase in the leakage current. Self-healing of the system is investigated during a period of 11 weeks after the delivery of the radiation dose. Annealing radiation-induced changes by bake-out at $100^{\circ} \mathrm{C}$ is investigated. It is concluded that the JUNGFRAU 1.0 pixel is sufficiently radiation-hard for its envisioned applications at SwissFEL and synchrotron beam lines. (C) 2015 AIP Publishing LLC. [http://dx.doi.org/10.1063/1.4938166]

\section{INTRODUCTION}

\section{A. Experimental situation/radiation environment at free electron lasers (FELs) and synchrotron light sources}

A new generation of large-scale X-ray sources, both FELs and synchrotrons, is becoming available to the photon science community shortly. ${ }^{1-9}$ These facilities are powerful, brilliant, and versatile in their performance characteristics and create a varied demand for adequate imaging detection systems for photon science at these light sources.

Key characteristics of these detection systems include among others ${ }^{10}$ single photon counting capabilities, a very large dynamic range ( 1 to tens of thousands of photons), a low false detection rate, a good spatial resolving power, a high quantum efficiency, a uniform gain response, and high frame rates. Large areas of detectors with small pixel sizes are required, gap-less detector geometries and vacuum compatibility are desirable detector features for high quality detection.

\footnotetext{
a) Author to whom correspondence should be addressed. Electronic mail: jsmith@magnet.fsu.edu.

b) Current address: National High Magnetic Field Laboratory, 1800 E. Paul Dirac Drive, Tallahassee, Florida 32310, USA.
}

Currently, FELs deliver about $10^{12}-10^{13}$ photons per pulse to the sample under investigation, which may result in signals of $10^{5}-10^{6}$ photons per pulse and per detector pixel during a single acquisition frame (originating, for instance, from a Bragg spot of a (nano)crystal) ${ }^{11}$ Due to the bunched time structure of FELs, these photons are delivered to the detection system in pulses as short as a few tens of femtoseconds. Synchrotrons continuously deliver $10^{12}$ or more photons per second to the sample under study, which may also result in diffraction peaks or scattered signal of $10^{6}-10^{7}$ photons $/ \mathrm{s}^{12}$ At the same time, significantly weaker signals are required to be registered accurately, i.e., weaker diffracting samples at larger diffraction angles result in signals of few or individual photons per pulse and detector pixel. A large dynamic range, on the order of $1-10^{4}$ photons is required. Importantly, a bright signal in one pixel should not corrupt low or no signal in neighboring pixels.

Varied photon occupation on the detector may result in the detection system being exposed to high local absorbed doses in consecutive measurements in particular if the detector is kept stationary throughout the measurements. ${ }^{13}$ The radiation delivered to the detection system may alter the detector hardware, induce degradation in the detector performance, and reduce the homogeneity of the detector response. 


\section{B. Effects of radiation on hybrid pixel detectors}

Radiation-induced effects, i.e., radiation damage, in hybrid silicon pixel detectors can be distinguished into effects on the sensor and effects on the application-specific integrated circuit (ASIC). ${ }^{14-16}$ In general, the effects on the sensor comprise bulk and surface defects. Bulk damage includes the displacement of crystal atoms, which alters the sensor's electrical properties. Surface damage includes changes in the covering dielectrics and in the interface region where the increase in the oxide charge represents the most prominent contribution. For X-rays, however, only surface effects are present. Sensor damage typically manifests itself as an increased leakage (translating in an increased noise) and charge trapping. The effects on the ASIC mainly comprise radiation-induced transistor parameter shifts which affect the analog frontend and appear as changes in the threshold voltages, an altered gain or speed or increased source-to-drain leakage. This type of radiation damage can in part be prevented by radiation tolerant layout-techniques and production processes. ${ }^{17}$

Spontaneous annihilation of radiation-induced damage by material self-healing, i.e., (partial) recovery of the original performance at normal operating conditions, is observed in silicon. ${ }^{18}$ Key factors in the recovery of the material are, for instance, hydrogen, oxygen, and silicon diffusion to anneal interface states, $\mathrm{SiO}_{2}$ bulk defects or $\mathrm{SiO}_{2}$ charge, and $\mathrm{Si}$ point defects. While some of these processes occur at room temperature (spontaneous healing), others require significantly higher temperatures of at least $80^{\circ} \mathrm{C}$ to $>300^{\circ} \mathrm{C}$ depending on the underlying physical process. Bake-out of radiation damaged $\mathrm{Si}$ detectors typically induces these recovery processes and is commonly performed to return towards or reestablish pre-irradiation performance. For instance, $60 \%$ of oxide-trapped charge gets released during a 15 -h bake-out at $100{ }^{\circ} \mathrm{C}$, while $80 \%$ are de-trapped during an equivalent 60 -h annealing bake-out. ${ }^{19,20}$

Though consideration is given to radiation-hard design techniques and an appropriate choice of production process for JUNGFRAU (adJUstiNg Gain detector FoR the Aramis User station), the work presented here is focused on studying as well as quantifying the effect of radiation damage on this hybrid pixel detector rather than unraveling the fundamental mechanisms of radiation damage.

The experimental requirements for photon science detection systems developed for experiments at new generation synchrotron and FEL light sources are introduced. The range of effects of radiation on hybrid pixel detectors is outlined (Section I). Section II describes the JUNGFRAU 1.0 hybrid pixel detector, expected radiation doses to the system at SwissFEL, and the design of the radiation hardness experiments. The effect of different radiation doses from 100 Gy to 10 MGy on detector noise, baseline, gain, and gain switching capabilities are investigated for both the bare ASIC and the hybridized assembly (Section III). The spontaneous recovery of the radiation-induced effects are studied throughout 11 weeks post-irradiation and the effect of detector bake-out on the detector performance/recovery is shown (Section IV). Section V concludes the study with suggestions for possible remedies for radiation-induced change, suggestions for further studies and an outlook.

\section{EXPERIMENTAL}

\section{A. JUNGFRAU 1.0 hybrid pixel detector}

JUNGFRAU is a hybrid pixel detector for photon science applications at free electron lasers and synchrotron light sources. ${ }^{21-23}$ JUNGFRAU is produced in $110 \mathrm{~nm}$ UMC (United Microelectronics Corporation) technology. Major characteristics of the JUNGFRAU charge-integrating pixel detector are single photon sensitivity and a low noise performance over a dynamic range of $10^{4} \cdot 12 \mathrm{keV}$ photons. These specifications are enabled by a gain-switching preamplifier with three gains in each pixel, which dynamically adjusts its gain to the amount of charge deposited on the pixel (similar to AGIPD $^{24}$ or GOTTHARD ${ }^{25}$ ). Performance characteristics of the JUNGFRAU chips also include a linearity and a gain uniformity to within a few percent and a high spatial resolving power. ${ }^{21-23}$

Geometrically, a JUNGFRAU chip measures about $2 \times 2 \mathrm{~cm}^{2}$ and comprises $256 \times 256$ pixels of $75 \times 75 \mu \mathrm{m}^{2}$ each. The chips are bump bonded to $320 \mu \mathrm{m}$ thick silicon sensors. $2 \times 4$ chips are tiled to form modules of $4 \times 8 \mathrm{~cm}^{2}$. Several multi-module systems with up to 16 megapixels per system are planned for the two end stations at SwissFEL and synchrotron beamlines. The anticipated readout rate in excess of $2 \mathrm{kHz}$ is independent of the detector size and enables a dead-time free, linear count rate capability of $20 \mathrm{MHz} /$ pixel (50 MHz/pixel) for $12 \mathrm{keV}(5 \mathrm{keV})$ photons, and meets both the readout requirements of SwissFEL and high count rate synchrotron experiments. ${ }^{21-23}$

\section{B. Expected irradiation for JUNGFRAU 1.0}

The radiation requirements for JUNGFRAU 1.0 are estimated based on the anticipated radiation rates at an experimental station at SwissFEL. In particular, the radiation requirements for experimental station $\mathrm{A}^{26}$ at the Aramis beamline of SwissFEL are considered where among others, a 16 megapixel JUNGFRAU system (=32 JUNGFRAU modules) will be installed. It is assumed that the experimental setup, in which the 16 megapixel JUNGFRAU detection system is integrated, will be used approximately $25 \%$ of the days per year. The system will be exposed to radiation $50 \%$ of the time during a 12-h shift per day. This results in a radiation exposure during approximately $2 \times 10^{6} \mathrm{~s} / \mathrm{yr}$. SwissFEL will deliver about $10^{10}$ photons per pulse at $100 \mathrm{~Hz}$, which results in $10^{12}$ photons/s. Accordingly, about $2 \times 10^{18}$ photons will be involved in experiments "around" JUNGFRAU per year. Assuming a fortunate crystal diffraction efficiency of $10^{-3}$, about $2 \times 10^{15}$ photons will impinge on the JUNGFRAU detector per year of operation.

FEL beam energies between 2 and $15 \mathrm{keV}$ are assumed. Homogeneous illumination throughout the 32 detector modules is considered. Also, an inhomogeneous illumination is examined where the dose is exclusively 
delivered to 4 detector modules, which corresponds to $12.5 \%$ of the detector pixels receiving the entire dose. Based on the detector material, the beam energy $(2-15 \mathrm{keV})$, and the expected photon fluence, we anticipate a dose to the detection system during $10 \mathrm{yr}$ of SwissFEL operation of $<20 \mathrm{kGy}$ for the homogeneous illumination and $<200 \mathrm{kGy}$ for a spatially more concentrated illumination. The dose is calculated as the amount of energy from ionizing radiation deposited in the mass of a certain material, i.e., dose $=$ absorbed energy $/$ mass $=\mu_{\mathrm{en}} / \rho \cdot \mathrm{N} \cdot \mathrm{E}$ in the unit of $1 \mathrm{~Gy}=1 \mathrm{~J} / \mathrm{kg}=100 \mathrm{rad}$, where $\mu_{\mathrm{en}} / \rho$ is the mass energy absorption coefficient, $\mathrm{N}$ is the photon fluence, and $\mathrm{E}$ is the energy per photon. ${ }^{15}$ These dose values represent the dose delivered to the surface of the hybrid pixel detector. The dose affecting the ASIC is reduced by the absorption of the photons in the $320 \mu \mathrm{m}$ Si sensor.

Similar doses are expected in the synchrotron environment ${ }^{13}$ where hybrid pixel detectors have been established as working-horse detection/imaging systems for many applications ranging from protein crystallography, powder diffraction to tomography. ${ }^{27}$ The single photon counting detection systems, PILATUS, for instance, were tested for radiation doses up to $30 \mathrm{kGy}^{13}$ and have successfully sustained the radiation doses delivered in synchrotron beamlines during periods of time in excess of 10 years.

These values compare to anticipated absorbed doses of 1 GGy during 3 years of operation for a detection system at the EU-XFEL, Hamburg, Germany ${ }^{11}$ or 5-10 MGy for the phase II upgrade of the Linear Hadron Collider (LHC), Geneva, Switzerland.

It should be noted that inadvertent, direct FEL or synchrotron beam irradiation delivers very high photon numbers on a few pixels of the system. This quickly leads to permanent if not fatal damage to the pixels caused by the delivered heat rather than (partially) reversible radiation damage through the mechanisms described in Section I B. This scenario and the associated doses are not considered in this study.

A dose <1 MGy is expected to be delivered to JUNGFRAU at FELs and synchrotron light sources. In the scope of this radiation hardness study, doses from $100 \mathrm{~Gy}$ up to doses in excess of $10 \mathrm{MGy}(=1 \mathrm{Grad})$ are delivered to JUNGFRAU to ensure a sound understanding of the radiationinduced effects on the detection system within and more than one order of magnitude beyond the expected dose range at SwissFEL.

\section{Radiation hardness study}

A specialized JUNGFRAU 1.0 assembly is constructed for the radiation hardness study to investigate the effects of radiation to the ASIC itself and the hybridized assembly, i.e., the ASIC bump-bonded to a Si sensor, separately. Radiationinduced effects on the ASIC and the sensor may be compared and disentangled in this way. Figure 1 shows a photograph of the custom assembly, in which a Si sensor is bump-bonded to the ASIC with an offset of 40 rows, i.e., 216 rows of the JUNGFRAU pixel matrix are covered by the Si sensor and 40 rows remain bare. The bare chip and the hybridized part of the

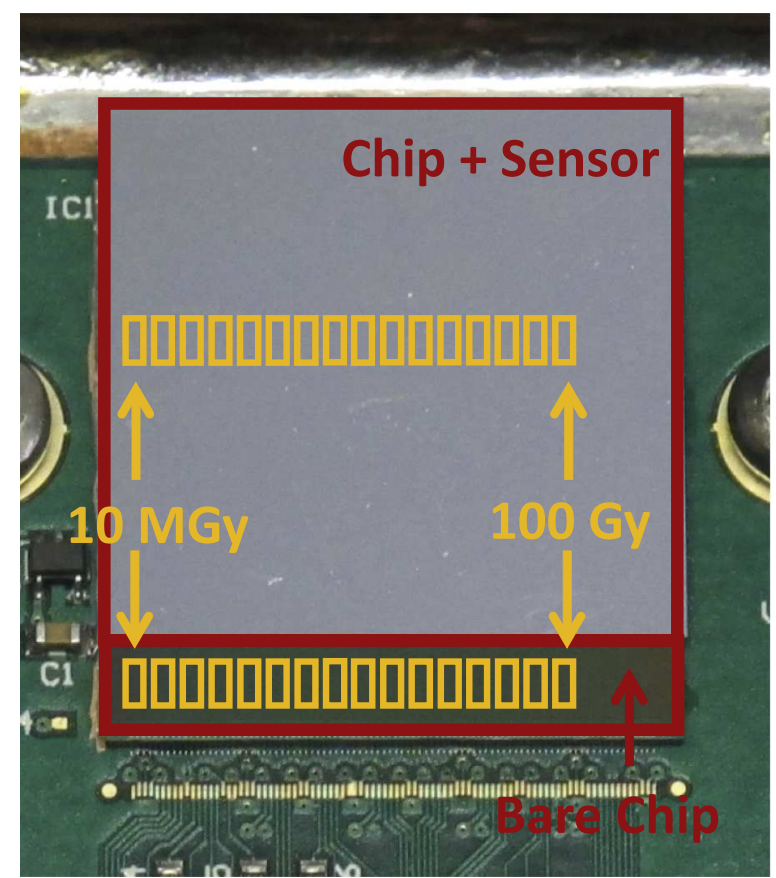

FIG. 1. Photograph of the JUNGFRAU 1.0 assembly for the radiation hardness tests. The Si sensor is offset by 40 rows. The bare chip and the chip bump-bonded to a $\mathrm{Si}$ sensor are both irradiated in 16 areas of approximately $7 \times 11$ pixels (indicated by the orange squares).

chip are each irradiated in 16 spots. Doses of approximately 100 Gy, 200 Gy, 500 Gy, 1 kGy, 2 kGy, 5 kGy, 10 kGy, 20 kGy, 50 kGy, 100 kGy, 200 kGy, 500 kGy, 1 MGy, 2 MGy, $5 \mathrm{MGy}$, and $10 \mathrm{MGy}$ are delivered to the ASIC in these areas. Note that the quoted doses are the doses actually received by the ASIC for both the bare and the sensor-covered parts of the assembly, i.e., the hybridized part of the assembly is irradiated longer in order to compensate for the photon intensity loss in the sensor medium.

All dose measurements up to $5 \mathrm{MGy}$ are conducted at the Nanoscopium beamline ${ }^{28}$ at the SOLEIL Synchrotron, Gifsur-Yvette Cedex, France. The two irradiation measurements at 10 MGy are performed at the OPTICS beamline ${ }^{29}$ at the Swiss Light Source, Paul Scherrer Institute, Villigen, Switzerland. The assembly is irradiated while powered and run at standard operating conditions with a sensor bias of $120 \mathrm{~V}$ and at a detector (chip and sensor) operating temperature of $20^{\circ} \mathrm{C}$ (active cooling). A photon beam energy of $15 \mathrm{keV}$ is selected for all radiation hardness measurements.

At the Nanoscopium beamline, the photon beam is collimated to cover a $7 \times 11$ pixels area on the detector. The photon flux is monitored throughout the measurement by a calibrated diode with a measurement uncertainty of less than $15 \%$ (uncertainty mainly due to thickness variations of the diode material), which introduces an equivalent uncertainty in the delivered dose. The doses from $100 \mathrm{~Gy}$ to $5 \mathrm{MGy}$ are delivered by exposing the chip area to the photon beam during an amount of time ranging from a fraction of a second (at $100 \mathrm{~Gy}$ ) to $>4 \mathrm{~h}$ (at $5 \mathrm{MGy}$ ). At the OPTICS beamline, a focused beam is available for the irradiation measurements. Hence, the two irradiation areas are successively stepped through the approximately $70 \times 140 \mu \mathrm{m}^{2}$ beam focus in 
several overlapping steps during a 3-day irradiation period to deliver a dose of more than $10 \mathrm{MGy}$ to the ASIC.

The setup of this radiation hardness study aims to mimic the irradiation of a JUNGFRAU 1.0 system during more than a decade of operation. Though equivalent radiation doses are delivered, there are few differences between the "real life scenario" and these tests at synchrotron light sources. Importantly, the presented experiments are conducted within minutes, hours, or few days, while the dose at the experimental stations will be delivered over a period of several years of operation. Accordingly, damage annihilation and self-healing of the material most likely takes place to a larger degree in the true experimental scenario and cannot be accounted for in the presented measurements. Also, synchrotron radiation is delivered continuously, while JUNGFRAU will among others serve at FELs where photons are delivered in short femtosecond pulses. The radiation-induced effects of short, intense photon pulses as opposed to steady photon irradiation might require further study.

\section{DETECTOR CHARACTERIZATION POST-IRRADIATION}

The JUNGFRAU 1.0 assembly is fully characterized in terms of noise, baseline, and gain pre-irradiation. This detailed performance evaluation is repeated post-irradiation. The pre- and post-irradiation performance is compared. The measurements are carried out in high gain mode, the sensor is biased at $120 \mathrm{~V}$, and the assembly is operated at $28^{\circ} \mathrm{C}$. Unless stated otherwise, the performance characteristic under investigation is obtained from the distribution of the respective values of all pixels irradiated by the same dose, i.e., a given performance characteristic is the mean of the distribution based on measurements from about 75 pixels, while the error in this value is given by the variance of this distribution.

Generally, all pixels are functional post-irradiation. However, some pixels irradiated with a dose of $10 \mathrm{MGy}$ are not capable of gain switching directly after irradiation but regained the gain switching capability spontaneously within a few hours post exposure.

\section{A. Baseline}

The level of the baseline of the irradiated pixels is investigated one week post-irradiation and compared to the pre-irradiation values. Figure 2 shows baseline of the full pixel matrix of the irradiated assembly for acquisition times of $2 \mu \mathrm{s}$ (Figure 2(a)), $20 \mu \mathrm{s}$ (Figure 2(b)), and $200 \mu \mathrm{s}$ (Figure 2(c)) in analog-to-digital converter units (ADCu). No significant change in the baseline is observed for delivered doses $\leq 5 \mathrm{MGy}$ on the bare chip (for all acquisition times). A slight baseline increase (corresponding to $3 \%-7 \%$ of the available range) is found for a delivered dose of $10 \mathrm{MGy}$ for the bare chip irradiation at all acquisition times. For the hybridized assembly instead, a rise in the baseline level is clearly present for delivered doses $>5 \mathrm{kGy}$. This indicates that the elevated baseline level mainly originates from a (a)

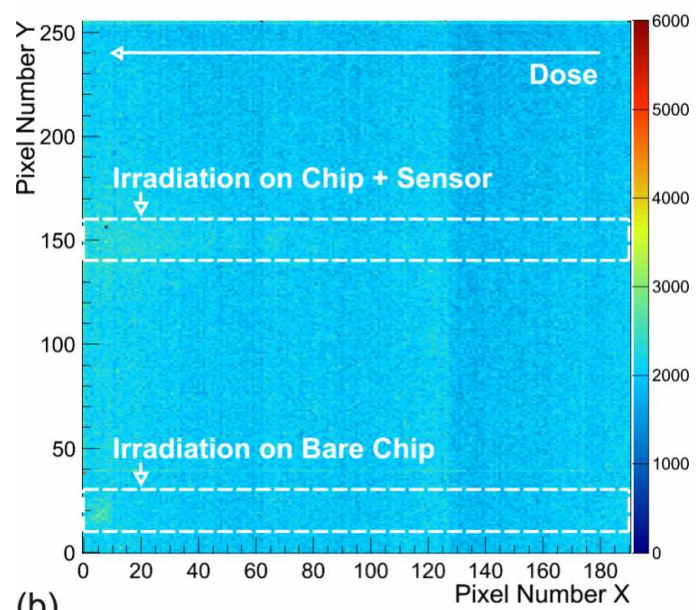

(b)

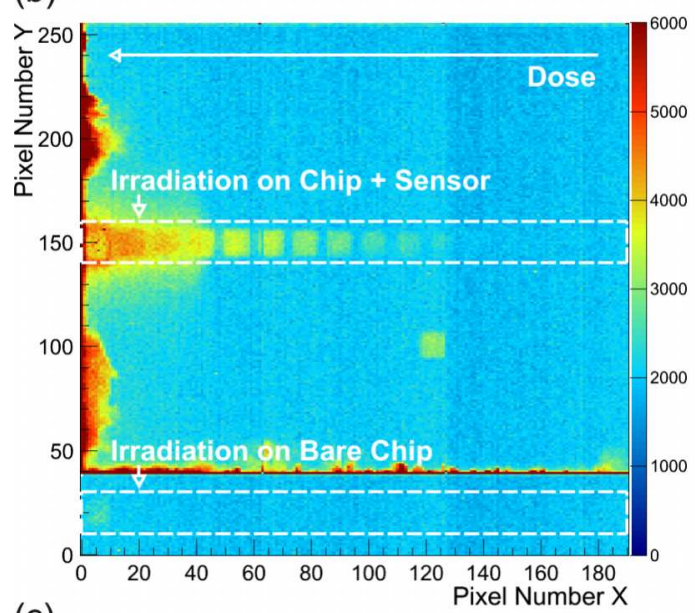

(c)

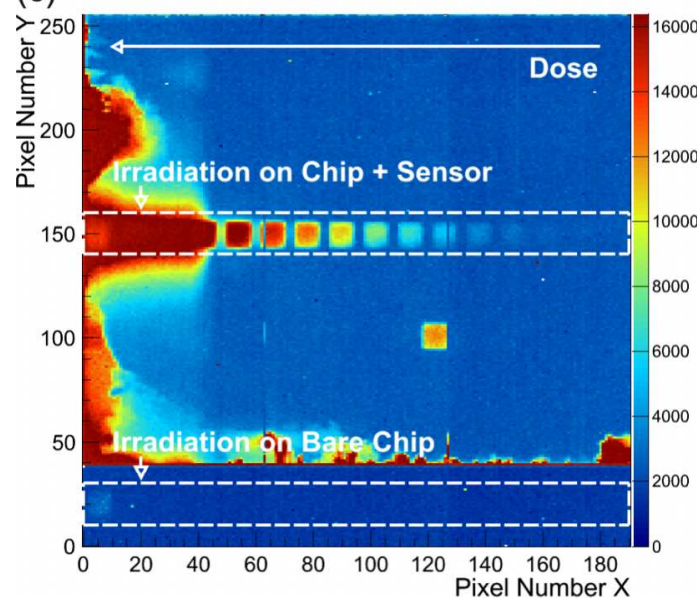

FIG. 2. Baseline of the JUNGFRAU 1.0 chip 1 week post-irradiation for acquisition times of (a) $2 \mu \mathrm{s}$, (b) $20 \mu \mathrm{s}$, and (c) $200 \mu \mathrm{s}$. Columns 192 through 255 are not displayed since they are not involved in the irradiation study. Note the different maxima of the color scale. The irradiated spot around pixel position $(120100)$ is caused by the detector alignment procedure.

radiation-induced increase in the sensor leakage current. The higher the delivered dose is, the more the baseline is raised. This effect is more pronounced for the longer integration times of $20 \mu \mathrm{s}$ and $200 \mu \mathrm{s}$ as expected. The origin of the "halo" surrounding higher doses in Figures 2(b) and 2(c) is not understood. 
An increase in the baseline level reduces the available detection range. The percentage reduction in the available range is given as reduction in range $(\%)=100 \% \cdot($ bbaseline $) /$ range $_{\text {pre-irradiation, }}$, where $\Delta$ baseline $=$ baseline $_{\text {post-irradiation }}$ - baseline $_{\text {pre-irradiation }}$ and range $_{\text {pre-irradiation }}=$ full range - baseline pre-irradiation.

Table I displays the percentage reduction in the available residual range in high gain mode due to the radiation-induced baseline shift for the bare chip and the hybridized assembly. Baseline fluctuation across the chip, which are already present pre-irradiation, are considered, and are eliminated in the postirradiation analysis of the baseline. As indicated in Figure 2, the range is unaffected for doses $\leq 5 \mathrm{MGy}$, while a reduction in the range of about $3 \%$ (for $2 \mu \mathrm{s}$ and $20 \mu \mathrm{s}$ acquisition times) and of about 7\% (for an acquisition time of $200 \mu \mathrm{s}$ ) is observed for the bare chip. For the hybridized assembly, the range is barely ( $\leq 2 \%$ and only $\geq 1 \mathrm{MGy}$ ) and somewhat $(\leq 15 \%$ for $\geq 10 \mathrm{kGy}$ ) reduced at acquisition times of $2 \mu \mathrm{s}$ (typical for FEL operation) and $20 \mu \mathrm{s}$, respectively. The hybridized assembly significantly loses range for the longer acquisition time of $200 \mu \mathrm{s}$. The range is reduced by $8 \%$ for a delivered dose of $1 \mathrm{kGy}$ up until $100 \%$, i.e., the baseline shift saturates the pixel at doses $\geq 1 \mathrm{MGy}$.

The post-irradiation measurements of the baseline indicate that the major contribution to the baseline shift is leakage current in the sensor since the induced dose does not show a significant effect on the baseline level in the bare ASIC. The radiation-induced increase in the leakage current and hence, baseline level is measured to be relatively small for acquisition times of $2 \mu \mathrm{s}$ and $20 \mu$ s, i.e., the reduction in the accessible range is $\leq 15 \%$ for typical FEL acquisition times. However, the baseline for the longer acquisition time of $200 \mu$ s is significantly shifted such that the range is reduced harshly ( $>33 \%$ for doses $\geq 10 \mathrm{kGy}$ ) or even completely diminished, i.e., the leakage current increase saturates the pixel $\geq 1$ MGy.

The observation that the baseline saturates for doses of 1 MGy and 5 MGy but does not saturate for a delivered dose of 10 MGy is compatible with prior investigations on high-dose X-ray radiation damage on silicon sensors. ${ }^{30,31}$ In particular, Ref. 31 shows a non-monotonic behavior of the oxide charge density and surface current density curves with a peak at around $1 \mathrm{MGy}$ as a function of the dose for the sensor material used in this work.

TABLE I. Percentage reduction in the range due to a radiation-induced baseline shift for the bare chip and the hybrid assembly.

\begin{tabular}{|c|c|c|c|c|c|c|c|}
\hline \multirow[b]{2}{*}{$\begin{array}{l}\text { Reduction in } \\
\text { range in high gain }\end{array}$} & \multirow[b]{2}{*}{$\begin{array}{l}\text { Acquisition } \\
\text { time }(\mu \mathrm{s})\end{array}$} & \multicolumn{6}{|c|}{ Dose delivered to ASIC (\%) } \\
\hline & & $\begin{array}{c}1 \\
\mathrm{kGy}\end{array}$ & $\begin{array}{c}10 \\
\mathrm{kGy}\end{array}$ & $\begin{array}{l}100 \\
\text { kGy }\end{array}$ & $\begin{array}{c}1 \\
\text { MGy }\end{array}$ & $\begin{array}{c}5 \\
\text { MGy }\end{array}$ & $\begin{array}{c}10 \\
\text { MGy }\end{array}$ \\
\hline \multirow{3}{*}{ Bare chip } & 2 & 0 & 0 & 0 & 0 & 0 & $\sim 3$ \\
\hline & 20 & 0 & 0 & 0 & 0 & 0 & $\sim 3$ \\
\hline & 200 & 0 & 0 & 0 & 0 & $\sim 2$ & $\sim 7$ \\
\hline \multirow{3}{*}{ Hybrid assembly } & 2 & 0 & 0 & 0 & $\sim 2$ & $\sim 2$ & $\sim 2$ \\
\hline & 20 & 0 & $\sim 4$ & $\sim 8$ & $\sim 12$ & $\sim 14$ & $\sim 15$ \\
\hline & 200 & $\sim 8$ & $\sim 33$ & $\sim 76$ & $\sim 100$ & $\sim 100$ & $\sim 86$ \\
\hline
\end{tabular}

The leakage current of the sensor can be reduced by cooling the system to lower operating temperatures. Figure 3 shows the baseline of the JUNGFRAU 1.0 assembly when cooled to $-10^{\circ} \mathrm{C}$. For the bare chip, the available range is now reduced by about $2 \%$ or less in the irradiated spots, which is similar to operation at $28^{\circ} \mathrm{C}$. For the hybridized assembly, a large portion of the range is recovered at an operating temperature of $-10^{\circ} \mathrm{C}$ (Table II) as compared to

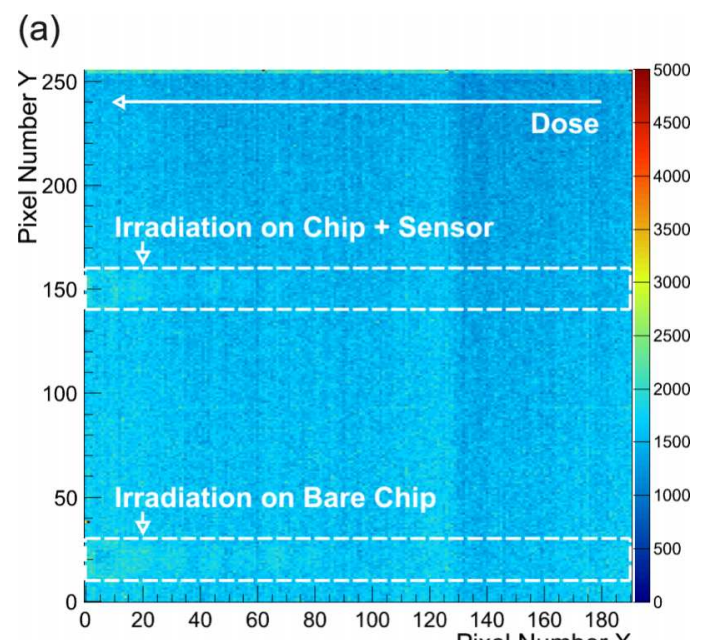

(b)
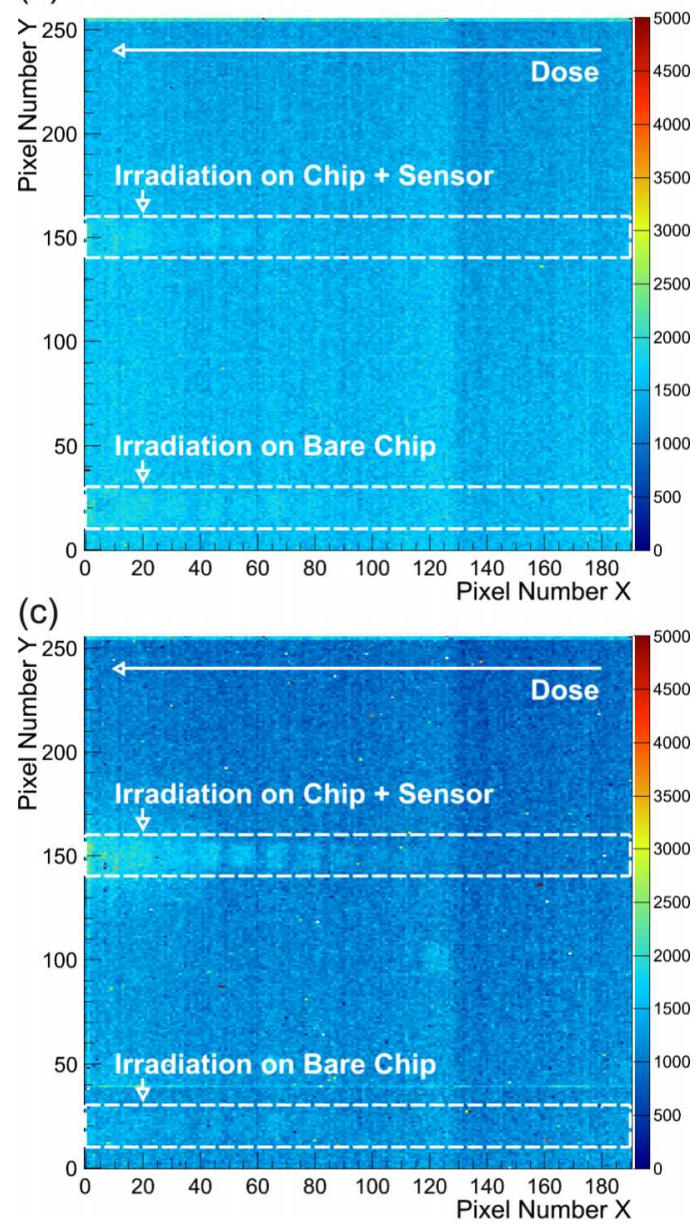

FIG. 3. Baseline of the JUNGFRAU 1.0 chip for acquisition times of (a) $2 \mu \mathrm{s}$, (b) $20 \mu \mathrm{s}$, and (c) $200 \mu \mathrm{s}$ with an assembly operating temperature of $-10^{\circ} \mathrm{C}$. Note the maximum of the color scale compared to Figure 2. 
TABLE II. Percentage reduction in the range due to a radiation-induced baseline shift for the bare chip and the hybrid assembly when operated at $-10^{\circ} \mathrm{C}$.

\begin{tabular}{|c|c|c|c|c|c|c|c|}
\hline \multirow[b]{2}{*}{$\begin{array}{l}\text { Reduction in } \\
\text { range in high gain }\end{array}$} & \multirow[b]{2}{*}{$\begin{array}{l}\text { Acquisition } \\
\text { time }(\mu \mathrm{s})\end{array}$} & \multicolumn{6}{|c|}{ Dose delivered to ASIC (\%) } \\
\hline & & $\begin{array}{c}1 \\
\mathrm{kGy}\end{array}$ & $\begin{array}{c}10 \\
\mathrm{kGy}\end{array}$ & $\begin{array}{l}100 \\
\mathrm{kGy}\end{array}$ & $\begin{array}{c}1 \\
\text { MGy }\end{array}$ & $\begin{array}{c}5 \\
\mathrm{MGy}\end{array}$ & $\begin{array}{c}10 \\
\text { MGy }\end{array}$ \\
\hline \multirow{3}{*}{ Bare chip } & 2 & 0 & 0 & 0 & 0 & 0 & 0 \\
\hline & 20 & 0 & 0 & 0 & 0 & 0 & 0 \\
\hline & 200 & 0 & 0 & 0 & 0 & $\sim 2$ & $\sim 2$ \\
\hline \multirow{3}{*}{ Hybrid assembly } & 2 & 0 & 0 & 0 & 0 & $\sim 2$ & $\sim 2$ \\
\hline & 20 & 0 & 0 & 0 & 0 & $\sim 2$ & $\sim 2$ \\
\hline & 200 & 0 & $\sim 2$ & $\sim 2$ & $\sim 5$ & $\sim 6$ & $\sim 7$ \\
\hline
\end{tabular}

operation at $28^{\circ} \mathrm{C}$. For acquisition times of $2 \mu$ s (Figure 3(a)) and $20 \mu$ s (Figure 3(b)), the range is practically unchanged for doses < $\mathrm{MGy}$ and reduced by about only $2 \%$ for higher doses (as compared to up to $15 \%$ for operation at $28^{\circ} \mathrm{C}$ ). For the $200 \mu$ s acquisition time (Figure 3(c)), the accessible range in the irradiated areas is reduced by less than $10 \%$, while the baseline is diminished by $33 \%-100 \%$, i.e., baseline saturation, when the assembly is operated at $28{ }^{\circ} \mathrm{C}$. As expected, the reduction in the operation temperature of the assembly by active cooling lowers the leakage current and returns the assembly into a good operating regime. Therefore, acquisition times on the order of $200 \mu \mathrm{s}$, which are customary for synchrotron applications, can also be enabled if cooling to $-10{ }^{\circ} \mathrm{C}$ can be provided.

Another future remedy to prevent radiation-induced baseline shifts and an according reduction in the detectable range could be the optimization of the sensor layout and/or geometry specifically for good radiation hardness and low leakage current such that usage at synchrotron light sources is facilitated.

\section{B. Noise}

The noise of the assembly is measured one week postirradiation at acquisition times of $2 \mu \mathrm{s}, 20 \mu \mathrm{s}$, and $200 \mu \mathrm{s}$. The pixel noise is calculated as the variance of a Gaussian fit to the pedestal, i.e., the pixel output signal in the absence of photon signal. Flat-field X-ray fluorescence measurements deliver photon spectra and are employed to determine the gain of each pixel in units of eV/ADC (analog-to-digital converter units). The relationship of $3.6 \mathrm{eV} /$ electron-hole pair in silicon sensors is used to convert the noise values into the equivalent noise charge (e.n.c.) in the unit of $\mathrm{e}^{-}$. The noise level of the hybridized assembly in $\mathrm{e}^{-}$is calculated using the according pixel gain after irradiation for each dose spot (Section III C).

Figure 4 shows the noise of the (a) bare ASIC in ADC units and (b) the hybridized assembly as a function of the delivered doses. The bare chip noise is unaltered for doses $<200 \mathrm{kGy}$ for all acquisition times. The noise in ADC units changes by up to $-20 \%$ for all acquisition times for doses between $200 \mathrm{kGy}$ and $10 \mathrm{MGy}$. This is probably due to a reduction in the gain.
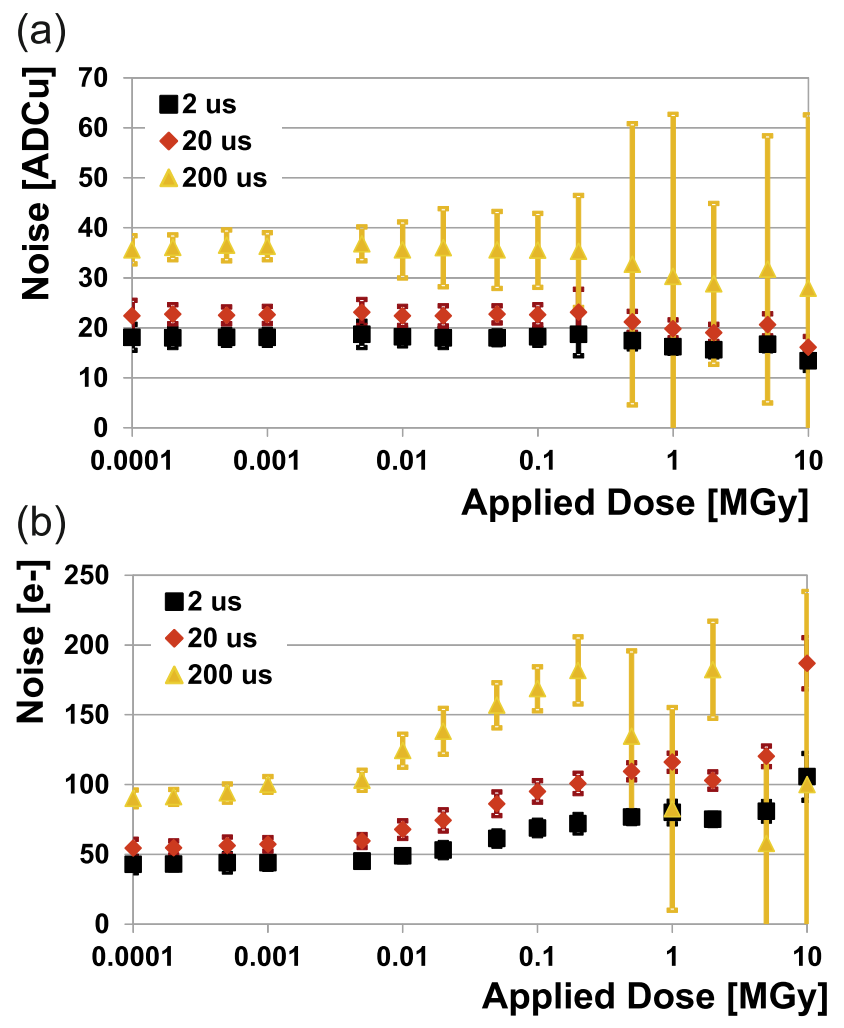

FIG. 4. JUNGFRAU 1.0 e.n.c. noise as a function of the delivered dose. (a) The noise of the bare ASIC in units of ADUu. (b) The noise of the hybridized assembly in units of $\mathrm{e}^{-}$.

The noise level of the hybridized assembly is unchanged for doses below $10 \mathrm{kGy}$. For higher doses, the noise level then increases from $40 \mathrm{e}^{-}$to $105 \mathrm{e}^{-}$, from $55 \mathrm{e}^{-}$to 185 $\mathrm{e}^{-}$, and from $90 \mathrm{e}^{-}$to $180 \mathrm{e}^{-}$for acquisition times of $2 \mu \mathrm{s}$, $20 \mu \mathrm{s}$, and $200 \mu \mathrm{s}$, respectively, which is mainly caused by the radiation-induced increase in the sensor leakage current. The discontinuity observed in the noise behavior for delivered doses $\geq 1 \mathrm{MGy}$ (for $200 \mu$ s acquisition times) correlates with the regime in which the JUNGFRAU 1.0 baseline is (almost) saturated due to radiation-induced effects (Figure 2). This noise plot looks qualitatively equivalent when displayed in $\mathrm{ADCu}$, except for the noise at a delivered dose of $10 \mathrm{MGy}$, which is altered by the change in gain (Section III C). The average gain conversion factor before irradiation is 0.47 $\mathrm{ADCu} / \mathrm{e}^{-}$.

For both the bare chip and the hybridized assembly, the variance between pixels in the mean noise value increases for doses $\geq 500 \mathrm{kGy}$ at acquisition times of $200 \mu \mathrm{s}$, which may be attributed to increased leakage in the pixel circuit that manifests itself more strongly for longer acquisition times.

\section{Gain}

The gain of JUNGFRAU 1.0 is evaluated one week post-irradiation. The previously dosed assembly is flat-field illuminated with $\mathrm{Cu} \mathrm{X}$-ray fluorescence photons generated by illuminating a $\mathrm{Cu}$ fluorescence target with a laboratory X-ray tube (General Electric Company, Seifert ID 3003 and analytical X-ray tube, $30 \mathrm{kV}, 45 \mathrm{~mA}$ ) at an acquisition time of 


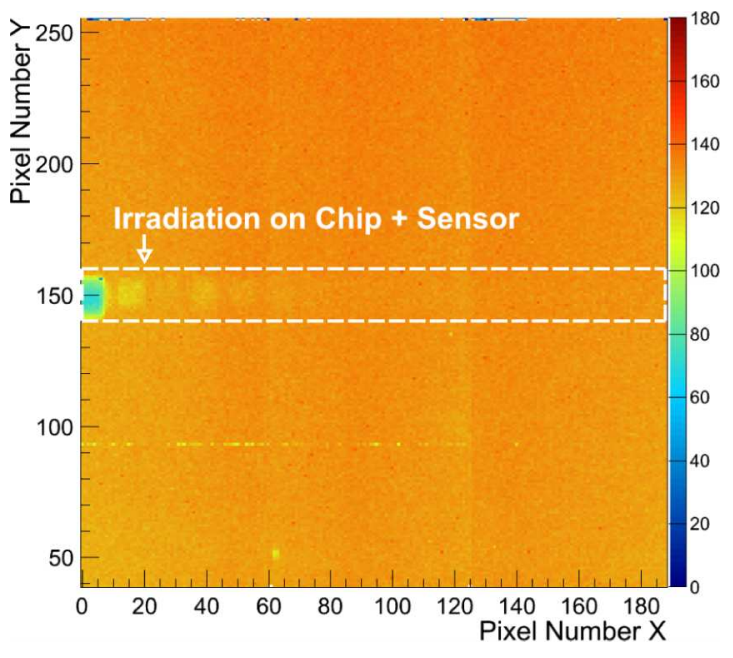

FIG. 5. JUNGFRAU 1.0 gain map post-irradiation in $\mathrm{ADCu} / \mathrm{keV}$ (hybridized part of the assembly only).

$2 \mu \mathrm{s}$. The resulting photon spectra are employed to determine the gain of each pixel in units of $\mathrm{eV} / \mathrm{ADCu}$.

Figure 5 shows the gain map of JUNGFRAU 1.0 after irradiation. Figure 6 displays the gain as a function of the delivered dose. Doses $\leq 500 \mathrm{kGy}$ do not influence the gain of the hybrid assembly, while a dose of 5 MGy and 10 MGy deteriorates the gain by about $10 \%$ and more than $50 \%$, respectively. The underlying mechanism for the decrease in the gain is currently not understood. The reduction in the gain reinforces the increase in the noise level for these delivered doses as observed in Figure 4(b).

\section{Effect of radiation on pixel preamplifier versus readout chain}

As observed in Secs. III A-III C, the main radiationinduced effect on the JUNGFRAU 1.0 detection system is the increase in the sensor leakage current (which manifests itself in an increased noise level) and a drop in the pixel gain as a function of the delivered dose. Therefore, it is interesting to understand where in the ASIC relevant changes are induced. It is important to realize if the radiation affects the preamplifier or the storage/readout chain of the pixel. ${ }^{21}$

Exclusively, the irradiated bare chip is considered. In particular, the pixel electronics after the preamplifier is tested

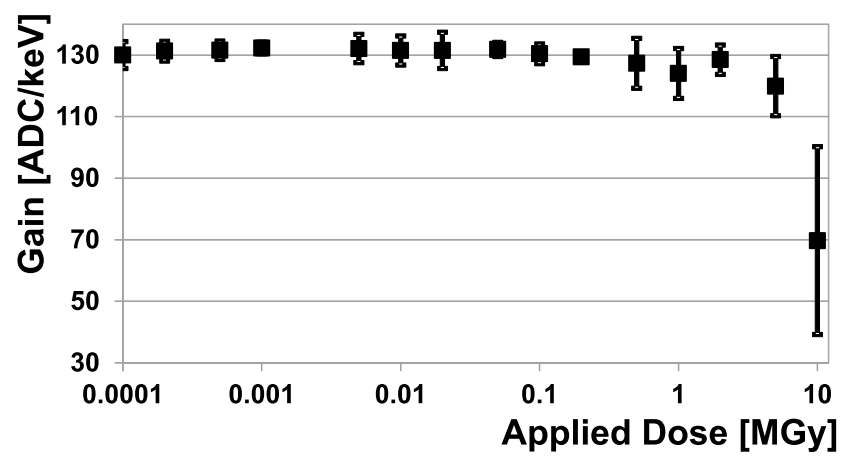

FIG. 6. JUNGFRAU 1.0 gain as a function of the delivered dose. to determine if this part of the circuit is affected by the delivered dose. For this test, the reference voltage of the correlated double sampling stage ${ }^{21}$ is directly injected to the storage cell and its value is scanned to simulate the signal generated by photon hits in a controlled way. The level of the baseline is recorded as a function of this reference voltage pre- and post-irradiation. It is observed that the level of the baseline scales perfectly linearly with the value of the reference voltage both before and after irradiation, i.e., the delivered dose does not affect the pixel readout circuit. In particular, the output curves of the pixel readout before and after irradiation display identical slopes and linearity. This indicates that the loss in gain (Section III C) does not originate from the pixel storage/readout chain but rather from the pixel preamplifier.

\section{RECOVERY OF THE RADIATION-INDUCED CHANGES}

\section{A. Spontaneous annealing}

The performance characteristics of the irradiated JUNGFRAU 1.0 assembly are monitored during 74 days post-irradiation. During this time, the irradiated assembly was stored in a standard laboratory environment at room temperature. In particular, the noise and the gain are measured in regular intervals after the delivery of the dose to study the spontaneous recovery of the radiation-induced damage.

Figure 7 displays the recovery of the noise of the bare ASIC (a) and the hybridized JUNGFRAU 1.0 assembly (b) for an acquisition time of $2 \mu \mathrm{s}$. The radiation-induced changes in the noise level return towards the pre-irradiation condition (bare chip noise $=18 \mathrm{ADCu}$ for an acquisition time of $2 \mu \mathrm{s}$ ) for the bare ASIC. In particular, the noise increases towards pre-irradiation levels during the recovery period, especially for delivered doses of 5 MGy and 10 MGy. Higher delivered doses reduce the noise more which is associated with the previously introduced reduction in the pixel gain. The changes induced in the hybridized assembly (given in the unit of $\mathrm{e}^{-}$) also gradually recover towards pre-irradiation levels (hybridized chip noise level about $45-50 \mathrm{e}^{-}$for an acquisition time of $2 \mu \mathrm{s}$ ). Qualitatively similar behavior is observed for the measurements at acquisition times of $20 \mu \mathrm{s}$ and $200 \mu \mathrm{s}$.

Figures 7(c) and 8 illustrate the spontaneous recovery of the detector gain towards pre-irradiation conditions. Figure 7(c) demonstrates the average recovery of the gain as a function of time for doses of $100 \mathrm{kGy}, 1 \mathrm{MGy}, 5 \mathrm{MGy}$, and 10 MGy. Since delivered doses $<10$ MGy only induce relatively small changes in the pixel gain, the recovery of the pixels dosed with $10 \mathrm{MGy}$ is most pronounced. Figure 8 shows single pixel spectra recorded with a pixel which has been irradiated with $10 \mathrm{MGy}$. The figure includes a pre-irradiation spectrum (black) and a spectrum recorded 2 days post-irradiation (red), which demonstrate that the radiation-induced effects have reduced the gain of the pixel by about $50 \%$. Also, spectra from 8 days, 16 days, 30 days, 47 days, 60 days, and 74 days post-irradiation are included, in which the pixel gain gradually recovers towards pre-irradiation conditions. 
(a)

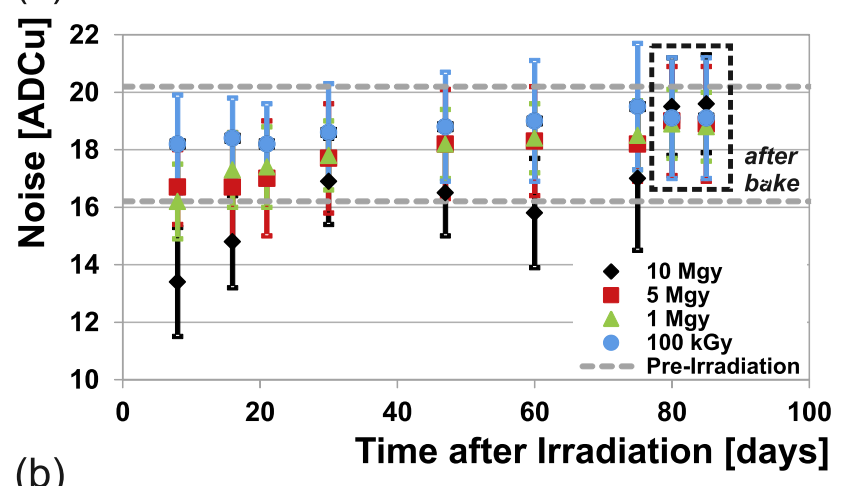

(b)

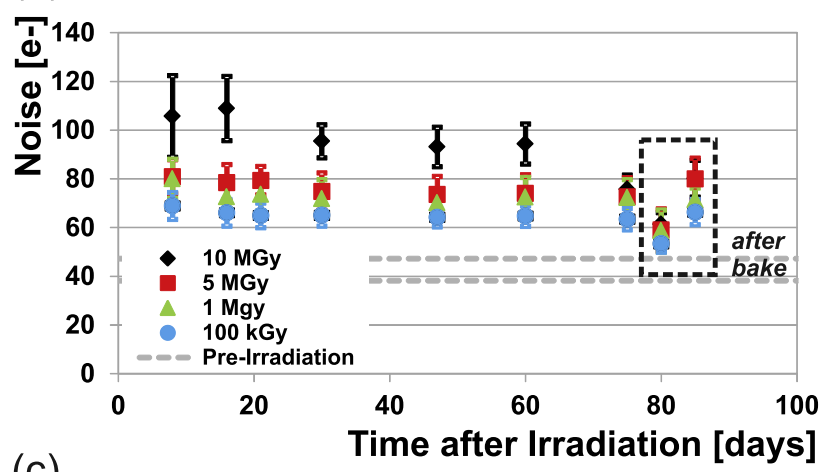

(c)

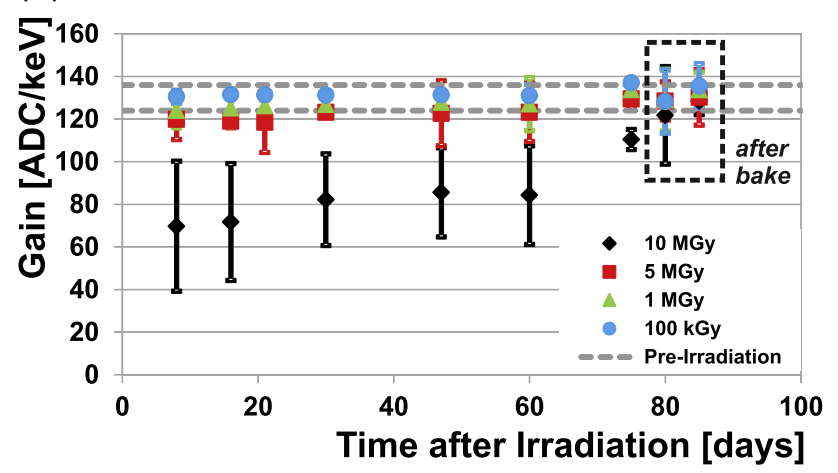

FIG. 7. JUNGFRAU 1.0 noise and gain recovery as a function of time after irradiation. (a) Noise of the bare chip, (b) noise of the hybridized assembly, and (c) gain of the hybridized assembly. The spontaneous recovery of the assembly during a period of 74 days post-irradiation is shown. The noise and gain after a $100^{\circ} \mathrm{C}$ bake-out of $15 \mathrm{~h}(80$ days post-irradiation $)$ and $60 \mathrm{~h}$ (85 days post-irradiation), respectively, are included (dashed boxes and Section IV B). The dashed grey lines indicate the pre-irradiation noise/gain values. The third data point (after 21 days) is inadvertently not collected for a dose of 10 MGy.

\section{B. Bake-out}

The irradiated JUNGFRAU 1.0 assembly is baked out 80 days post-irradiation to initiate further annealing of the radiation-induced effects on the assembly. The complete assembly is heated to $100{ }^{\circ} \mathrm{C}$ in an oven for a period of $15 \mathrm{~h}$ (bake-out 1) and $60 \mathrm{~h}$ (bake-out 2), respectively. Though some radiation-induced effects only anneal at temperatures $>300^{\circ} \mathrm{C}$ (Section I B), a maximum baking temperature of $100^{\circ} \mathrm{C}$ is chosen since several components of the hybridized assembly cannot withstand significantly higher temperatures. No bias is applied during baking.

The assembly is characterized after each bake-out step (Figure 7, black dashed boxes). The largest effect of the

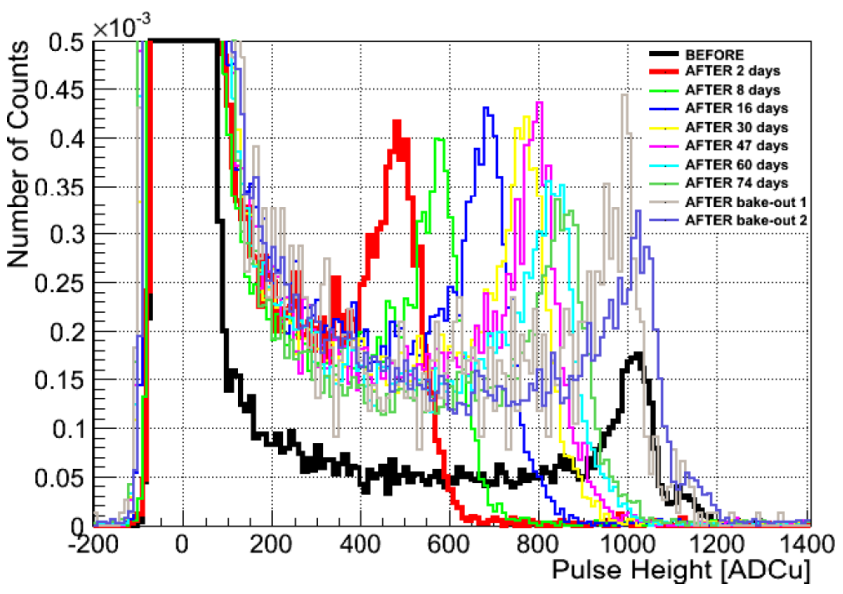

FIG. 8. JUNGFRAU 1.0 single pixel spectrum of $\mathrm{Cu}$ fluorescence before irradiation (black) and directly after irradiation (red) with a dose of $10 \mathrm{MGy}$ is shown. Spectra at several points in time during a recovery period of 74 days post-irradiation are displayed. Two spectra after a 15 -h (bake-out 1 ) and a 60 -h (bake-out 2) bake-out at $100^{\circ} \mathrm{C}$ are included.

bake-out is observed for pixels dosed with 10 MGy since the most significant changes in the pixel noise and gain is introduced by this dose. After bake-out, the noise of the bare chip returns to its pre-irradiation value. The noise of the hybridized assembly appears to be reduced (closer to the pre-irradiation value) after the first bake-out of $15 \mathrm{~h}$ and increased again after the 60 -h bake-out. The gain of the assembly is found to have increased towards and returned to the pre-irradiation values by the 15 -h and 60 -h bake-out, respectively (Figure 8).

\section{v. CONCLUSIONS AND OUTLOOK}

This work evaluates the radiation hardness of the JUNGFRAU 1.0 hybrid pixel detector for photon science at FELs and synchrotron light sources. Doses up to10 MGy are delivered to the JUNGFRAU pixel matrix during this study, while maximally doses on the order of $200 \mathrm{kGy}(<1 \mathrm{MGy})$ are expected to be delivered to the system during 10 years of routine operation at SwissFEL end stations and synchrotron beamlines.

Importantly, the JUNGFRAU 1.0 pixel can sustain doses of 10 MGy. Performance parameters like the baseline level, the noise of the hybridized assembly, and the pixel gain are affected by the absorbed doses: The level of the baseline as well as the noise of the hybridized assembly increases, while the gain decreases for doses $\geq 500 \mathrm{kGy}$. Mainly, the leakage current of the sensor is affected by the delivered dose, which increases the noise and the baseline level of the pixels. Also, the preamplifier, rather than the storage/readout chain of the pixel, is responsible for the changed gain of the circuit. For acquisition times of $2 \mu \mathrm{s}$ and $20 \mu \mathrm{s}$, the radiation induced effects are minimal and can easily be accommodated. For an acquisition time of $200 \mu \mathrm{s}$, the baseline is affected significantly by the delivered dose such that additional measures, i.e., an optimized, more radiation hard sensor or stringent cooling of the detector assembly might become necessary. The radiation induced effects (partially) recover spontaneously 
post-irradiation and can further be recovered via a bake-out of the assembly.

In the future, further experiments to study the effect of radiation on the pixel periphery, in particular off-chip drivers and current mirrors, ${ }^{21}$ are planned to complement the presented study which is focused on the radiation hardness of the JUNGFRAU 1.0 pixel.

\section{ACKNOWLEDGMENTS}

The research leading to these results has received funding from the European Community's Seventh Framework Programme (FP7/2007-2013) under Grant Agreement No. 290605 (PSI-FELLOW/COFUND). Part of the experimental work presented in this article was conducted at the OPTICS beam line and precursor studies were performed at the MicroXAS beamline, Swiss Light Source, Villigen PSI, Switzerland. The authors acknowledge the support of the OPTICS and the MicroXAS beam line staff in particular Uwe Flechsig, Daniel Grolimund, Vallerie Ann Samson, Beat Meyer, and Dario Ferreira Sanchez. We thank Chris Milne and Jakub Szlachetko of the experimental station A of SwissFEL for fruitful discussion on expected radiation doses. The authors are grateful to the NANOSCOPIUM beamline staff of the SOLEIL Synchrotron, Gif-sur-Yvette Cedex, France, in particular the beamline responsible, Andrea Somogyi, to allocate the beamtime to us and Gil Baranton for his technical support.

${ }^{1}$ B. D. Patterson, R. Abela, H. H. Braun, U. Flechsig, R. Ganter, Y. Kim, E. Kirk, A. Oppelt, M. Pedrozzi, and S. Reiche, New J. Phys. 12, 035012 (2010).

${ }^{2}$ SwissFEL Conceptual Design Report, edited by R. Ganter, Paul Scherrer Institut, 2010.

${ }^{3}$ D. Pile, Nat. Photonics 5(8), 456 (2011).

${ }^{4}$ J. Arthur, G. Materlik, R. Tatchyn, and H. Winick, Rev. Sci. Instrum. 66(2), 1987 (1995).

${ }^{5}$ J. Arthur, U. Bergmann, A. Brunger, C. Bostedt, S. Boutet, J. Bozek, D. Cocco, T. Devereaux, Y. Ding, H. Dürr, D. Fritz, K. Gaffney, J. Galayda, J. Goldstein, J. Gühr, J. Hastings, P. Heimann, K. Hodgson, Z. Huang, N. Kelez, P. Montanez, A. Robert, M. Rowen, W. Schlotter, M. Seibert, J. Stöhr, J. Turner, W. White, J. Wu, T. Williams, V. Yachandra, and J. Yano, A White Paper Outlining Science and Scope of Instrumentation (2012).

${ }^{6}$ EU-XFEL, DESY, Hamburg, Germany, 2015.

${ }^{7}$ ESRF Upgrade Programme, Phase II (2015-2022): Technical design study, edited by G. Admans, P. Berkvens, A. Kaprolat, and J.-L. Revol (ESRF, 2014).

${ }^{8}$ M. Ehrlichman, M. Aiba, M. Böge, A. Saa-Hernandez, and A. Streun, in The XXII European Synchrotron Light Source Workshop (ESLS), Grenoble, France, 2014.
${ }^{9}$ P. F. Tavares, S. C. Leemann, M. Sjöström, and Å. Andersson, J. Synchrotron Radiat. 21, 862 (2014).

${ }^{10}$ Synchrotron Radiation News 27(4) (2014) [Issue on Photon Detectors].

${ }^{11}$ H. Graafsma, J. Instrum. 4, P12011 (2009).

${ }^{12}$ G. Hülsen, Ch. Broennimann, E. F. Eikenberry, and A. Wagner, J. Appl. Crystallogr. 39, 550 (2006).

${ }^{13}$ B. A. Sobott, Ch. Broennimann, E. F. Eikenberry, R. Dinapoli, P. Kraft, G. N. Taylor, P. R. Willmott, C. M. Schlepütz, and R. P. Sassool, J. Synchrotron Radiat. 16, 489 (2009).

${ }^{14}$ L. Rossi, P. Fischer, T. Rohe, and N. Wermes, Pixel Detectors: From Fundamentals to Application (Springer, 2006).

${ }^{15}$ W. R. Leo, Techniques for Nuclear and Particle Physics Experiments: A How-to Approach (Springer Verlag Berlin Heidelberg GmbH, 1994).

${ }^{16} \mathrm{G}$. Lindström, Nucl. Instrum. Methods Phys. Res., Sect. A 512, 30 (2003).

${ }^{17}$ L. Gonella, F. Faccio, M. Silvestri, S. Erardin, D. Pantano, V. Re, M. Manghisoni, L. Ratti, and A. Ranieri, Nucl. Instrum. Methods Phys. Res., Sect. A 582, 750-754 (2007).

${ }^{18}$ J. Schmitz, Self-Healing in Semiconductors for Radiation Hardness, International Workshop on Radiation Imaging and Detection (IWoRID, Hamburg, Germany, 2015).

${ }^{19}$ F. Faccio and G. Cervelli, IEEE Trans. Nucl. Sci. 52, 6 (2005).

${ }^{20}$ L. Dusseault, T. L. Randolph, R. D. Schrimpf, K. F. Galloway, F. Saigné, J. Fesquet, J. Gasiot, and R. Ecoffet, J. Appl. Phys. 81, 2437 (1997).

${ }^{21}$ A. Mozzanica, A. Bergamaschi, S. Cartier, R. Dinapoli, D. Greiffenberg, I. Johnson, J. Jungmann, D. Maliakal, D. Mezza, Ch. Ruder, L. Schaedler, B. Schmitt, X. Shi, and G. Tinti, J. Instrum. 9, C05010 (2014).

${ }^{22}$ J. H. Jungmann-Smith, A. Bergamaschi, S. Cartier, R. Dinapoli, D. Greiffenberg, I. Johnson, D. Maliakal, D. Mezza, A. Mozzanica, Ch. Ruder, L. Schaedler, B. Schmitt, X. Shi, and G. Tinti, J. Instrum. 9, P12013 (2014).

${ }^{23}$ A. Mozzanica, A. Bergamaschi, M. Brueckner, S. Cartier, R. Dinapoli, D. Greiffenberg, J. H. Jungmann-Smith, D. Maliakal, D. Mezza, M. Ramilli, Ch. Ruder, L. Schaedler, B. Schmitt, X. Shi, and G. Tinti, "Characterization results of the JUNGFRAU full scale readout ASIC," J. Instrum. (submitted).

${ }^{24}$ P. Goettlicher, H. Graafsma, H. Hirsemann, S. Jack, B. Nilsson, G. Potdevin, I. Sheviakov, F. Tian, U. Trunk, C. Youngman, M. Zimmer, J. Becker, E. Fretwurst, R. Klanner, H. Perrey, I. Pintilie, A. K. Srivastava, R. Dinapoli, B. Henrich, A. Mozzanica, B. Schmitt, X. Shi, M. Karagounis, and H. Kruger, IEEE Nucl. Sci. Symp. Conf. Rec. 1817 (2009).

${ }^{25}$ A. Mozzanica, A. Bergamaschi, R. Dinapoli, H. Graafsma, D. Greiffenberg, B. Henrich, I. Johnson, M. Lohmann, R. Valeria, B. Schmitt, and S. Xintian, J. Instrum. 7, C01019 (2012).

${ }^{26}$ C. J. Milne, SwissFEL Experimental Station A: Conceptual Design Report, 2013.

${ }^{27}$ A. Bergamaschi, S. Cartier, R. Dinapoli, D. Greiffenberg, J. H. Jungmann-Smith, D. Mezza, A. Mozzanica, B. Schmitt, X. Shi, and G. Tinti, J. Instrum. 10, C01033 (2014).

${ }^{28}$ A. Somogyi, K. Medjoubi, G. Baranton, V. Le Roux, M. Ribbens, F. Polack, P. Philippot, and J.-P. Samam, J. Synchrotron Radiat. 22, 1118 (2015).

${ }^{29}$ U. Flechsig, A. Jaggi, S. Spielmann, H. A. Padmore, and A. A. MacDowell, Nucl. Instrum. Methods Phys. Res., Sect. A 609, 281 (2009).

${ }^{30}$ R. Klanner, E. Fretwurst, I. Pintilie, J. Schwandt, and J. Zhang, "Study of high-dose X-ray radiation damage of silicon sensors," Nucl. Instrum. Methods Phys. Res., Sect. A 732, 117-121 (2013).

${ }^{31}$ J. Zhang, E. Fretwurst, H. Graafsma, R. Klanner, I. Kopsalis, and J. Schwandt, "Study of X-ray radiation damage in the AGIPD sensor for the European XFEL," J. Instrum. 9, C05022 (2014). 